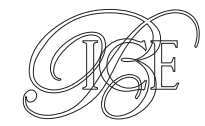

\title{
6. Inversiones exteriores
}


6.1. DISTRIBUCIÓN POR PAÍSES DEL FLUJO DE INVERSIÓN BRUTA ESPAÑOLA EN EL EXTERIOR (EXCLUYENDO LAS ETVE)

(Millones de euros)

\begin{tabular}{|c|c|c|c|c|c|c|c|}
\hline & 2011 & 2012 & 2013 & 2014 & 2015 & 2016 & 2017 \\
\hline Estados Unidos de América ........................ & 3.141 & 751 & 800 & 3.247 & 1.302 & 5.091 & 2.801 \\
\hline 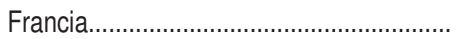 & 358 & 1.786 & 241 & 87 & 1.000 & 433 & 2.795 \\
\hline 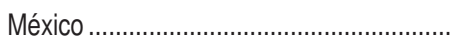 & 1.504 & 649 & 805 & 824 & 2.085 & 643 & 1.721 \\
\hline 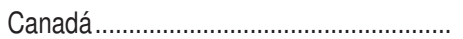 & 98 & 375 & 116 & 223 & 9.112 & 481 & 1.567 \\
\hline 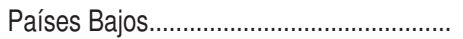 & 572 & 2.885 & 1.232 & 901 & 696 & 2.174 & 1.487 \\
\hline Colombia & 314 & 281 & 585 & 852 & 480 & 466 & 1.476 \\
\hline 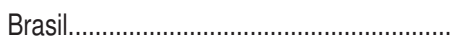 & 4.809 & 1.269 & 1.939 & 4.263 & 5.801 & 1.537 & 1.055 \\
\hline Portugal ................................................... & 370 & 346 & 358 & 813 & 399 & 452 & 975 \\
\hline 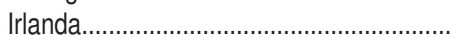 & 2.785 & 1.082 & 519 & 4.726 & 7 & 4.206 & 946 \\
\hline 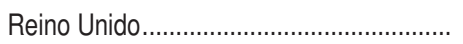 & 4.119 & 210 & 2.498 & 1.832 & 3.917 & 8.905 & 571 \\
\hline 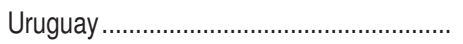 & 243 & 450 & 379 & 111 & 479 & 29 & 545 \\
\hline 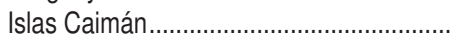 & 12 & 10 & 160 & 1.970 & 1 & 11 & 518 \\
\hline Perú & 91 & 351 & 4.843 & 391 & 263 & 396 & 378 \\
\hline 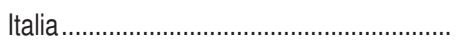 & 467 & 573 & 1.448 & 524 & 1.148 & 1.383 & 351 \\
\hline 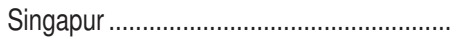 & 6 & 17 & 1 & 5 & 17 & 10 & 300 \\
\hline 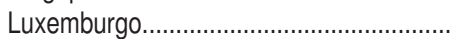 & 513 & 432 & 1.936 & 268 & 243 & 1.990 & 274 \\
\hline 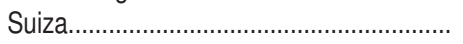 & 19 & 22 & 232 & 46 & 6 & 56 & 253 \\
\hline 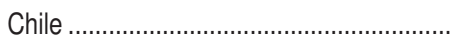 & 689 & 2.378 & 281 & 5.369 & 908 & 3.740 & 181 \\
\hline 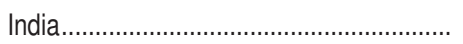 & 55 & 51 & 139 & 77 & 54 & 85 & 158 \\
\hline Argentina & 275 & 456 & 433 & 265 & 156 & 2.309 & 149 \\
\hline 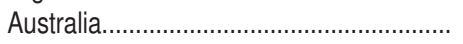 & 52 & 63 & 86 & 49 & 31 & 22 & 140 \\
\hline 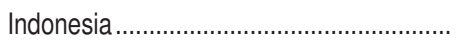 & 2 & 1 & 33 & 0 & 8 & 1 & 124 \\
\hline 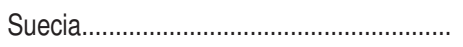 & 15 & 5 & 8 & 697 & 187 & 62 & 124 \\
\hline China & 631 & 194 & 203 & 549 & 252 & 156 & 95 \\
\hline 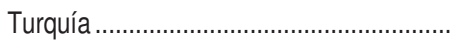 & 4.592 & 303 & 500 & 88 & 64 & 55 & 89 \\
\hline 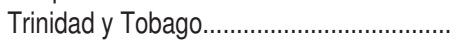 & 0 & 12 & 3 & 7 & 46 & 7 & 86 \\
\hline 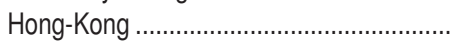 & 49 & 12 & 3 & 119 & 2 & 18 & 78 \\
\hline Hungría .................................................. & 53 & 7 & 7 & 81 & 14 & 4 & 68 \\
\hline 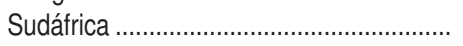 & 7 & 7 & 7 & 25 & 19 & 10 & 54 \\
\hline 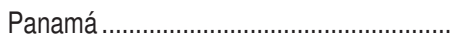 & 81 & 172 & 92 & 49 & 88 & 19 & 44 \\
\hline 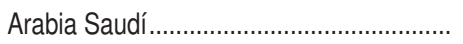 & 156 & 42 & 179 & 20 & 18 & 0 & 40 \\
\hline 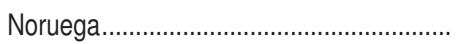 & 156 & 76 & 78 & 176 & 268 & 50 & 35 \\
\hline Venezuela.............................................. & 182 & 50 & 57 & 645 & 99 & 483 & 28 \\
\hline Nicaragua & 11 & 10 & 0 & 18 & - & 17 & 28 \\
\hline 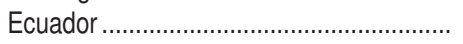 & 18 & 87 & 58 & 437 & 110 & 57 & 21 \\
\hline Paraguay & 50 & 13 & 5 & 1 & 1 & 23 & 19 \\
\hline 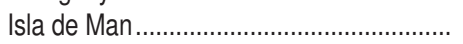 & - & 0 & - & 3 & - & 0 & 15 \\
\hline 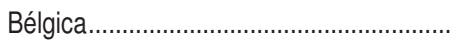 & 263 & 17 & 59 & 214 & 8 & 20 & 15 \\
\hline Malta................................................. & 29 & 42 & 8 & 3 & 36 & 5 & 14 \\
\hline 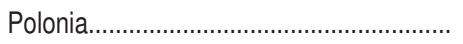 & 4.221 & 96 & 381 & 553 & 41 & 22 & 11 \\
\hline 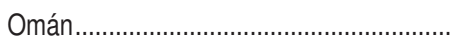 & 1 & & 4 & 6 & 10 & 14 & 10 \\
\hline 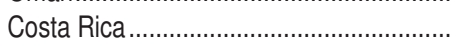 & 150 & 146 & 37 & 37 & 61 & 25 & 10 \\
\hline Austria & 25 & 167 & 101 & 8 & 549 & 31 & 10 \\
\hline 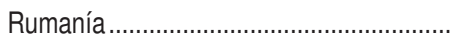 & 34 & 50 & 6 & 184 & 60 & 13 & 9 \\
\hline Cuba & 8 & 54 & 5 & - & - & 10 & 9 \\
\hline 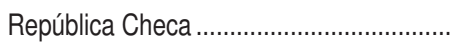 & 11 & 65 & 20 & 29 & 18 & 83 & 9 \\
\hline República Eslovaca .................................... & 8 & 0 & 27 & 4 & 3 & 4 & 8 \\
\hline 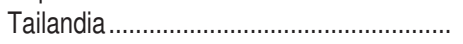 & 0 & 1 & 12 & 0 & 1 & 0 & 7 \\
\hline 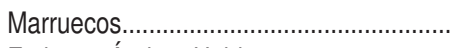 & 143 & 18 & 15 & 23 & 28 & 29 & 7 \\
\hline Emiratos Árabes Unidos............................... & 5 & 11 & 3 & 44 & 12 & 1 & 7 \\
\hline 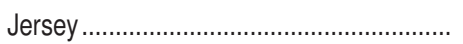 & 0 & 20 & 1 & 12 & 0 & 11 & 6 \\
\hline Guatemala & 13 & 174 & 3 & 54 & 9 & 15 & 6 \\
\hline 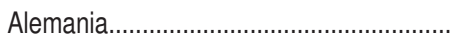 & 1.232 & 578 & 4.125 & 77 & 851 & 958 & 5 \\
\hline República Dominicana.............................. & 18 & 35 & 24 & 17 & 25 & 10 & 4 \\
\hline 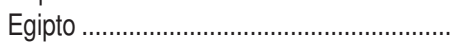 & 89 & 19 & - & 17 & 131 & 65 & 4 \\
\hline 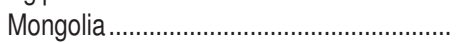 & - & 2 & 0 & - & 1 & - & 3 \\
\hline 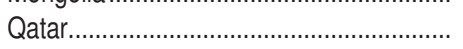 & 0 & 1 & 1 & 1 & 1 & 2 & 3 \\
\hline 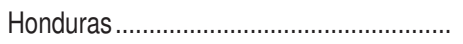 & 33 & 0 & - & 12 & 1 & 8 & 2 \\
\hline 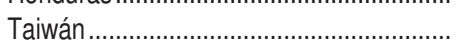 & 16 & 0 & - & - & 0 & 4 & 2 \\
\hline
\end{tabular}


6.1. DISTRIBUCIÓN POR PAÍSES DEL FLUJO DE INVERSIÓN BRUTA ESPAÑOLA EN EL EXTERIOR (EXCLUYENDO LAS ETVE) (continuación)

(Millones de euros)

\begin{tabular}{|c|c|c|c|c|c|c|c|}
\hline & 2011 & 2012 & 2013 & 2014 & 2015 & 2016 & 2017 \\
\hline Bolivia & 0 & 2 & 3 & 18 & 33 & 0 & 2 \\
\hline Islas Vírgenes Británicas ................................. & 27 & 3 & 0 & 36 & 58 & 3 & 1 \\
\hline 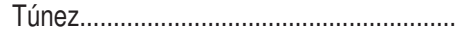 & 17 & 8 & 0 & 2 & 10 & 16 & 1 \\
\hline Barbados & - & - & 0 & 5 & & 0 & 1 \\
\hline 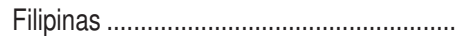 & 0 & - & 0 & 0 & 0 & 3 & 1 \\
\hline Croacia & 13 & 2 & 5 & 0 & 3 & 4 & 1 \\
\hline 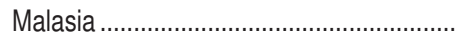 & 70 & 17 & 37 & 90 & 0 & 3 & 1 \\
\hline Rusia & 74 & 37 & 108 & 124 & 65 & 44 & 1 \\
\hline 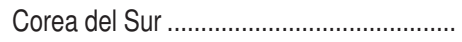 & 41 & 14 & 7 & 4 & 10 & 209 & 1 \\
\hline Mauricio & 17 & 9 & 53 & 49 & 16 & 25 & 0 \\
\hline 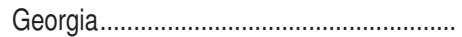 & - & 0 & 0 & - & - & - & 0 \\
\hline Andorra & 32 & 2 & 4 & 1 & 151 & 11 & 0 \\
\hline Vietnam & 0 & - & - & 0 & 0 & 0 & 0 \\
\hline Bahrein & 1 & 0 & - & - & - & 6 & 0 \\
\hline 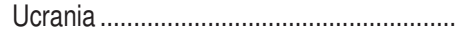 & 8 & 0 & 2 & 2 & 5 & 0 & 0 \\
\hline Bulgaria & 1 & 29 & 4 & 5 & 4 & 21 & 0 \\
\hline 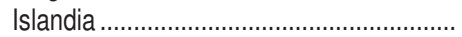 & 28 & 159 & - & - & - & - & 0 \\
\hline Serbia & 48 & 10 & 9 & 12 & 7 & 1 & 0 \\
\hline Angola & 19 & 1 & - & - & 1 & - & 0 \\
\hline Argelia & 11 & 13 & 3 & 9 & 37 & 12 & 0 \\
\hline Chipre & 18 & - & 63 & - & - & 4 & 0 \\
\hline 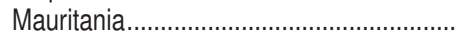 & 0 & 0 & 2 & 0 & 0 & - & 0 \\
\hline Grecia & 39 & 89 & 35 & 3 & 2 & 6 & 0 \\
\hline Benin & 0 & - & - & - & - & - & 0 \\
\hline 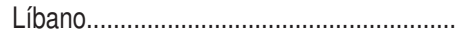 & 0 & 7 & - & 0 & - & - & 0 \\
\hline Guinea Ecuatorial ........................................... & 0 & 0 & 0 & 0 & 0 & - & 0 \\
\hline Subtotal & 33.257 & 17.328 & 25.436 & 31.411 & 31.531 & 37.079 & 19.761 \\
\hline Resto. & 173 & 529 & 459 & 299 & 145 & 183 & 0 \\
\hline TOTAL & 33.429 & 17.857 & 25.895 & 31.710 & 31.676 & 37.262 & 19.761 \\
\hline OCDE & 25.377 & 13.133 & 15.935 & 20.871 & 22.924 & 31.124 & 14.437 \\
\hline UE-15 & 10.759 & 8.170 & 12.589 & 10.158 & 9.054 & 20.661 & 7.553 \\
\hline UE-27 & 15.139 & 8.459 & 13.107 & 11.017 & 9.229 & 20.818 & 7.672 \\
\hline UE-28 ……………………… & 15.152 & 8.461 & 13.112 & 11.017 & 9.233 & 20.822 & 7.673 \\
\hline 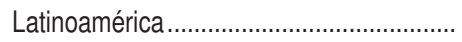 & 8.495 & 6.609 & 9.924 & 13.524 & 10.617 & 9.808 & 5.677 \\
\hline 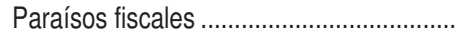 & 152 & 143 & 233 & 2.078 & 84 & 89 & 541 \\
\hline Europa & 20.070 & 9.139 & 14.037 & 11.471 & 9.801 & 21.050 & 8.073 \\
\hline 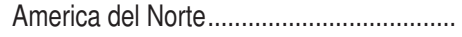 & 3.239 & 1.127 & 916 & 3.470 & 10.414 & 5.572 & 4.368 \\
\hline 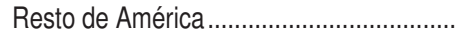 & 134 & 409 & 165 & 2.042 & 105 & 121 & 606 \\
\hline Asia y Oceanía & 1.101 & 460 & 753 & 1.006 & 439 & 535 & 969 \\
\hline 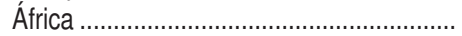 & 342 & 104 & 90 & 184 & 293 & 176 & 66 \\
\hline ASEAN & 79 & 36 & 84 & 96 & 26 & 17 & 433 \\
\hline MENA & 465 & 185 & 221 & 151 & 296 & 151 & 86 \\
\hline Centroamérica & 309 & 544 & 529 & 346 & 194 & 114 & 94 \\
\hline 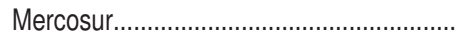 & 5.559 & 2.239 & 2.813 & 5.284 & 6.536 & 4.381 & 1.796 \\
\hline Alianza Pacífico & 2.597 & 3.659 & 6.513 & 7.436 & 3.737 & 5.245 & 3.756 \\
\hline Comunidad Andina & 423 & 721 & 5.489 & 1.698 & 886 & 919 & 1.876 \\
\hline CCG & 162 & 53 & 187 & 71 & 41 & 23 & 60 \\
\hline Caricom & 4 & 376 & 5 & 14 & 53 & 52 & 87 \\
\hline Latam & 8.492 & 6.584 & 9.922 & 13.522 & 10.610 & 9.808 & 5.677 \\
\hline 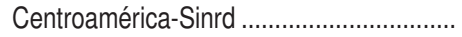 & 291 & 509 & 506 & 329 & 169 & 104 & 90 \\
\hline
\end{tabular}

Fuente: Registro de Inversiones Exteriores, Ministerio de Industria, Comercio y Turismo. 
6.2. DISTRIBUCIÓN POR PAÍSES DEL FLUJO DE INVERSIÓN BRUTA EXTRANJERA EN ESPAÑA (EXCLUYENDO LAS ETVE)

(Millones de euros)

\begin{tabular}{|c|c|c|c|c|c|c|c|}
\hline & 2011 & 2012 & 2013 & 2014 & 2015 & 2016 & 2017 \\
\hline 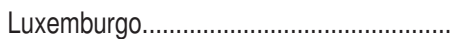 & 1.985 & 1.759 & 1.387 & 2.563 & 3.519 & 3.123 & 4.976 \\
\hline 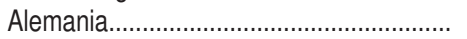 & 1.279 & 2.067 & 1.362 & 450 & 952 & 2.248 & 3.222 \\
\hline 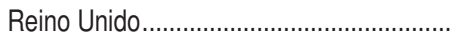 & 7.731 & 1.000 & 1.986 & 1.701 & 1.141 & 2.249 & 3.107 \\
\hline Estados Unidos de América ....................... & 1.619 & 2.298 & 2.311 & 3.793 & 2.670 & 5.110 & 2.550 \\
\hline 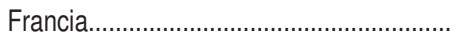 & 5.732 & 873 & 1.913 & 2.040 & 2.145 & 1.900 & 2.314 \\
\hline 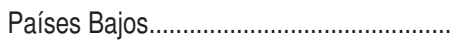 & 1.535 & 1.719 & 1.801 & 1.173 & 2.751 & 1.908 & 1.997 \\
\hline 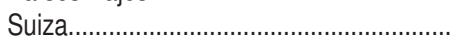 & 699 & 341 & 282 & 405 & 451 & 303 & 1.356 \\
\hline 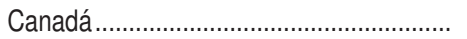 & 36 & 12 & 97 & 313 & 68 & 828 & 744 \\
\hline Мéxico & 75 & 152 & 390 & 1.214 & 951 & 1.372 & 454 \\
\hline 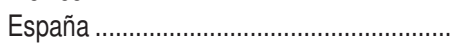 & 384 & 1.195 & 1.974 & 764 & 3.350 & 369 & 246 \\
\hline 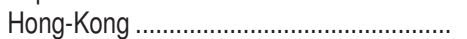 & 5 & 40 & 241 & 292 & 221 & 55 & 227 \\
\hline Italia & 319 & 194 & 261 & 106 & 158 & 139 & 222 \\
\hline Uruguay & 88 & 118 & 29 & 465 & 458 & 305 & 221 \\
\hline 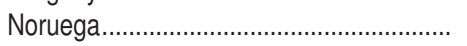 & 27 & 24 & 59 & 8 & 37 & 108 & 193 \\
\hline 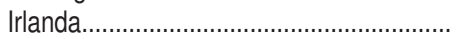 & 41 & 347 & 197 & 379 & 193 & 485 & 175 \\
\hline 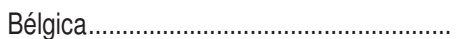 & 104 & 292 & 402 & 165 & 76 & 184 & 157 \\
\hline 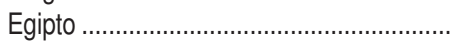 & 0 & 0 & 4 & 1 & 1 & 1 & 155 \\
\hline Singapur & 11 & 5 & 21 & 385 & 160 & 80 & 144 \\
\hline Sudáfrica & 0 & 0 & 4 & 0 & 16 & 12 & 138 \\
\hline Andorra & 191 & 39 & 108 & 77 & 44 & 62 & 126 \\
\hline China & 62 & 142 & 527 & 610 & 1.483 & 1.120 & 90 \\
\hline 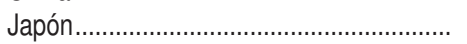 & 205 & 55 & 176 & 460 & 404 & 509 & 71 \\
\hline Perú & 1 & 48 & 2 & 9 & 88 & 2 & 65 \\
\hline 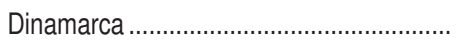 & 37 & 14 & 65 & 13 & 9 & 24 & 54 \\
\hline 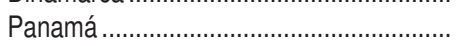 & 24 & 85 & 77 & 92 & 45 & 45 & 52 \\
\hline Argentina & 7 & 6 & 5 & 11 & 42 & 8 & 48 \\
\hline Chipre & 31 & 40 & 6 & 5 & 26 & 2 & 47 \\
\hline 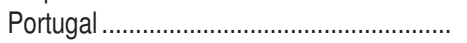 & 729 & 128 & 54 & 136 & 450 & 415 & 46 \\
\hline Chile & 5 & 14 & 10 & 63 & 17 & 160 & 42 \\
\hline 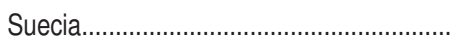 & 555 & 340 & 55 & 113 & 111 & 112 & 40 \\
\hline Israel............................................. & 11 & 20 & 14 & 212 & 33 & 25 & 37 \\
\hline 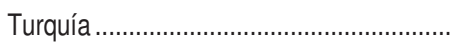 & 1 & 8 & 33 & 4 & 60 & 32 & 36 \\
\hline 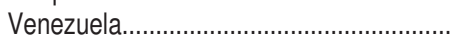 & 23 & 34 & 167 & 1.113 & 28 & 45 & 32 \\
\hline 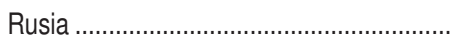 & 52 & 42 & 62 & 43 & 58 & 46 & 31 \\
\hline 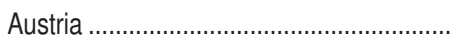 & 129 & 55 & 112 & 58 & 63 & 112 & 24 \\
\hline Corea del Sur & 1 & 312 & 46 & 48 & 13 & 108 & 24 \\
\hline 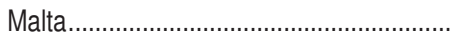 & 15 & 66 & 65 & 28 & 60 & 41 & 21 \\
\hline 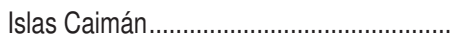 & 29 & 93 & 20 & 36 & 25 & 25 & 19 \\
\hline 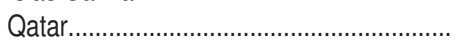 & 0 & 2 & 66 & 17 & 2 & 677 & 18 \\
\hline 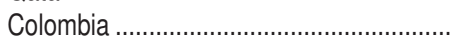 & 1 & 29 & 7 & 4 & 8 & 6 & 17 \\
\hline 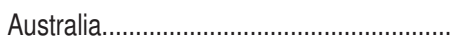 & 2 & 2 & 45 & 7 & 10 & 9 & 15 \\
\hline 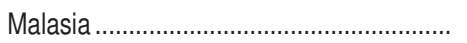 & - & 0 & 0 & 0 & 1 & 1 & 14 \\
\hline Brasil.................................................... & 25 & 245 & 209 & 225 & 226 & 193 & 13 \\
\hline 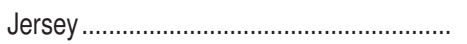 & 24 & 21 & 3 & 25 & 64 & 130 & 13 \\
\hline 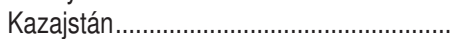 & 3 & 0 & 8 & 4 & 25 & 1 & 12 \\
\hline 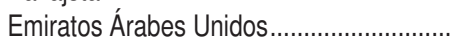 & 4.379 & 9 & 15 & 55 & 175 & 19 & 10 \\
\hline 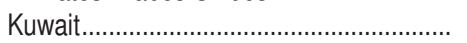 & 1 & 0 & 1 & 3 & 497 & 1 & 10 \\
\hline 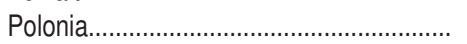 & 7 & 1 & 33 & 22 & 3 & 46 & 10 \\
\hline 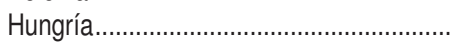 & 5 & 5 & 4 & 0 & 0 & 1 & 8 \\
\hline 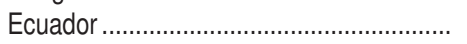 & 4 & 2 & 6 & 1 & 18 & 25 & 8 \\
\hline 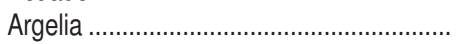 & 1 & 0 & 141 & 0 & 0 & 1 & 8 \\
\hline 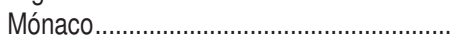 & 7 & 2 & 3 & 5 & 1 & 3 & 7 \\
\hline Islas Vírgenes Británicas .............................. & 37 & 22 & 11 & 10 & 6 & 2 & 7 \\
\hline 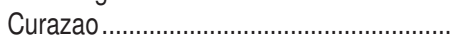 & - & - & 29 & 87 & 38 & 39 & 7 \\
\hline 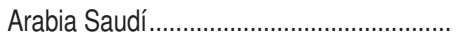 & 31 & 10 & 12 & 17 & 445 & 45 & 7 \\
\hline 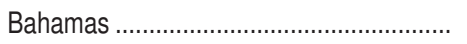 & 7 & 1 & 8 & 4 & 16 & 5 & 6 \\
\hline 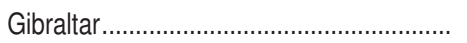 & 16 & 18 & 8 & 13 & 11 & 15 & 6 \\
\hline Liechtenstein & 14 & 11 & 5 & 6 & 288 & 14 & 6 \\
\hline 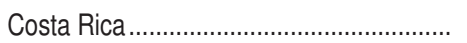 & 12 & 7 & 10 & 17 & 55 & 9 & 6 \\
\hline Ucrania & 0 & 0 & 9 & 5 & 1 & 11 & 5 \\
\hline
\end{tabular}


6.2. DISTRIBUCIÓN POR PAÍSES DEL FLUJO DE INVERSIÓN BRUTA EXTRANJERA EN ESPAÑA (EXCLUYENDO LAS ETVE) (continuación)

(Millones de euros)

\begin{tabular}{|c|c|c|c|c|c|c|c|}
\hline & 2011 & 2012 & 2013 & 2014 & 2015 & 2016 & 2017 \\
\hline 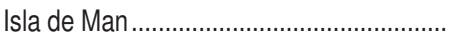 & 18 & 1 & 0 & 3 & - & 1 & 4 \\
\hline Finlandia & 106 & 6 & 11 & 3 & 7 & 3 & 4 \\
\hline 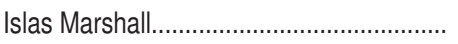 & - & 4 & - & 3 & 0 & - & 3 \\
\hline 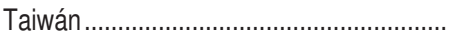 & 0 & 5 & 2 & 14 & 6 & 1 & 3 \\
\hline 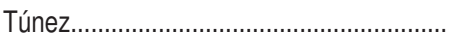 & 0 & 0 & 1 & 1 & 1 & 4 & 3 \\
\hline India & 28 & 103 & 6 & 1 & 15 & 5 & 2 \\
\hline Indonesia & - & 0 & 0 & 0 & 0 & 0 & 2 \\
\hline Guernsey & 29 & 8 & 0 & 316 & 1 & 6 & 2 \\
\hline 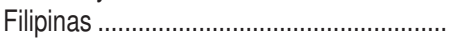 & 0 & 0 & 2 & 20 & 50 & 143 & 2 \\
\hline Namibia & - & - & - & 0 & 0 & 0 & 2 \\
\hline Siria & 4 & 0 & 2 & 0 & 0 & 5 & 1 \\
\hline 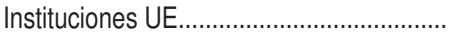 & - & - & - & - & - & - & 1 \\
\hline 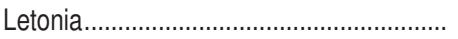 & 0 & 4 & 1 & 0 & 1 & 1 & 1 \\
\hline Rumanía & 4 & 2 & 0 & 0 & 0 & 0 & 1 \\
\hline 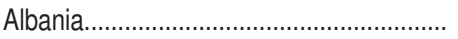 & 0 & - & 1 & - & 0 & 319 & 1 \\
\hline Líbano & 15 & 3 & 0 & 2 & 3 & 1 & 1 \\
\hline Iraq & - & 0 & 0 & 0 & 0 & 0 & 1 \\
\hline Barbados & 34 & 0 & 4 & 5 & 3 & 13 & 1 \\
\hline República Dominicana................................. & 1 & 2 & 0 & 3 & 0 & 16 & 1 \\
\hline 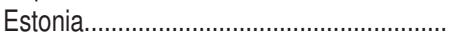 & 0 & 0 & 0 & 1 & 0 & 0 & 1 \\
\hline 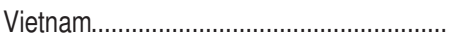 & 0 & - & - & - & - & 0 & 1 \\
\hline 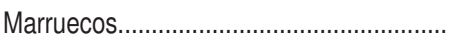 & 6 & 21 & 27 & 0 & 0 & 27 & 1 \\
\hline Bulgaria …...................................... & 1 & 0 & 1 & 0 & 1 & 1 & 1 \\
\hline 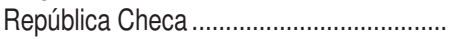 & 1 & 15 & 4 & 2 & 8 & 1 & 1 \\
\hline 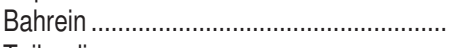 & - & 0 & 0 & - & 0 & 20 & 0 \\
\hline 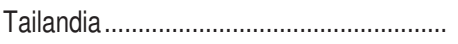 & 0 & 0 & 0 & 0 & 2 & 0 & 0 \\
\hline 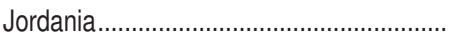 & 0 & 0 & 3 & 34 & 0 & 0 & 0 \\
\hline Lituania & 1 & 20 & 1 & 0 & 107 & 0 & 0 \\
\hline 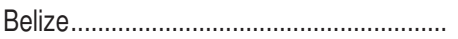 & 12 & 2 & 3 & 0 & 1 & 6 & 0 \\
\hline Grecia & 2 & 2 & 1 & 1 & 8 & 55 & 0 \\
\hline Angola & 0 & 0 & 0 & 0 & 5 & 1 & 0 \\
\hline 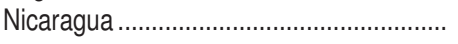 & - & - & 0 & 8 & - & - & 0 \\
\hline 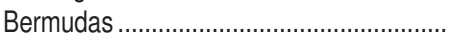 & 74 & 14 & 0 & 2 & 234 & 5 & 0 \\
\hline 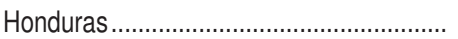 & - & 0 & - & - & - & 7 & 0 \\
\hline Cuba & 0 & 1 & 0 & 0 & 0 & 0 & 0 \\
\hline Guinea Ecuatorial & 2 & 2 & 0 & 0 & 1 & 0 & 0 \\
\hline 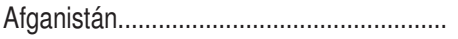 & - & - & 0 & 3 & 0 & 0 & 0 \\
\hline 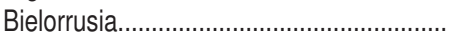 & 0 & 0 & 0 & 0 & 1 & 0 & 0 \\
\hline República de Cabo Verde ......................... & - & 0 & - & - & 0 & - & 0 \\
\hline Guatemala & 0 & 7 & 3 & 2 & 9 & 0 & 0 \\
\hline 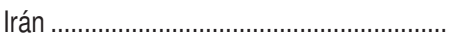 & 0 & 0 & 0 & 0 & 3 & 1 & 0 \\
\hline Georgia & 0 & 1 & - & 0 & - & 0 & 0 \\
\hline 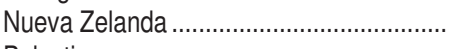 & 18 & 114 & 18 & 29 & 0 & 2 & 0 \\
\hline Palestina & - & - & - & - & 0 & - & 0 \\
\hline 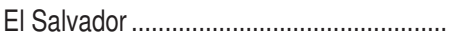 & 5 & 0 & 0 & 0 & 5 & 0 & 0 \\
\hline Mauricio & 0 & - & 96 & 27 & 1 & 0 & 0 \\
\hline Seychelles y dependencias ........................... & 0 & 0 & 0 & 0 & 0 & 0 & 0 \\
\hline Serbia & 0 & 0 & 0 & 0 & 0 & 0 & 0 \\
\hline 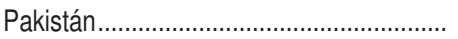 & 0 & 0 & 0 & 0 & 0 & 0 & 0 \\
\hline 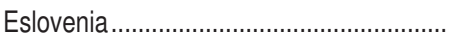 & 0 & 0 & 0 & 1 & 1 & 0 & 0 \\
\hline República Eslovaca ................................... & 0 & 0 & 1 & 1 & 0 & 0 & 0 \\
\hline Bolivia & 0 & - & - & - & 0 & 0 & 0 \\
\hline Libia & 0 & 0 & 0 & 0 & 0 & 2 & 0 \\
\hline 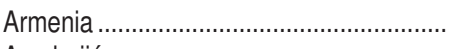 & 0 & - & 0 & 1 & 0 & 0 & 0 \\
\hline 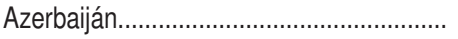 & - & 0 & 2 & - & 0 & 0 & 0 \\
\hline Nigeria & 0 & 0 & 0 & - & 4 & 1 & 0 \\
\hline 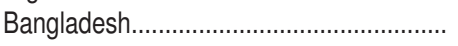 & 0 & - & 0 & 0 & 0 & 0 & 0 \\
\hline Islandia & 0 & 0 & 7 & - & 0 & 0 & 0 \\
\hline 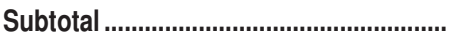 & 28.714 & 14.702 & 17.156 & 20.359 & 24.748 & 25.570 & 23.758 \\
\hline 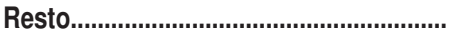 & 18 & 27 & 115 & 21 & 37 & 32 & 0 \\
\hline TOTAL_............................................................. & 28.732 & 14.729 & 17.271 & 20.380 & 24.785 & 25.602 & 23.758 \\
\hline
\end{tabular}


6.2. DISTRIBUCIÓN POR PAÍSES DEL FLUJO DE INVERSIÓN BRUTA EXTRANJERA EN ESPAÑA (EXCLUYENDO LAS ETVE) (continuación)

(Millones de euros)

\begin{tabular}{|c|c|c|c|c|c|c|c|}
\hline & 2011 & 2012 & 2013 & 2014 & 2015 & 2016 & 2017 \\
\hline OCDE ........ & 23.378 & 13.365 & 15.111 & 16.248 & 19.659 & 21.938 & 22.125 \\
\hline UE-15 ................ & 20.667 & 9.991 & 11.582 & 9.664 & 14.934 & 13.324 & 16.584 \\
\hline UE-27 & 20.732 & 10.145 & 11.697 & 9.724 & 15.141 & 13.418 & 16.674 \\
\hline 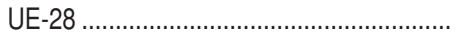 & 20.732 & 10.145 & 11.697 & 9.724 & 15.142 & 13.418 & 16.674 \\
\hline 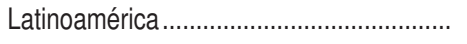 & 282 & 753 & 971 & 3.227 & 1.951 & 2.205 & 960 \\
\hline 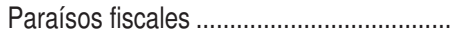 & 273 & 191 & 149 & 485 & 635 & 247 & 65 \\
\hline 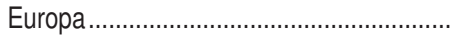 & 21.815 & 10.657 & 12.277 & 10.634 & 16.159 & 14.467 & 18.459 \\
\hline 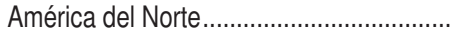 & 1.655 & 2.310 & 2.408 & 4.106 & 2.738 & 5.938 & 3.294 \\
\hline Resto de América ......................................... & 191 & 152 & 133 & 148 & 346 & 92 & 40 \\
\hline Asia y Oceanía .............................................. & 4.779 & 829 & 1.208 & 2.231 & 3.557 & 2.828 & 697 \\
\hline África & 11 & 28 & 274 & 34 & 35 & 72 & 307 \\
\hline ASEAN & 12 & 6 & 23 & 405 & 214 & 224 & 163 \\
\hline 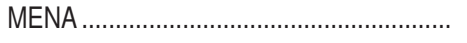 & 4.465 & 131 & 351 & 371 & 1.221 & 870 & 273 \\
\hline 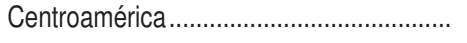 & 42 & 101 & 90 & 121 & 114 & 77 & 59 \\
\hline 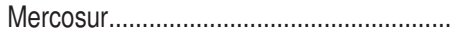 & 143 & 402 & 418 & 1.814 & 754 & 552 & 315 \\
\hline Alianza Pacífico ........................................... & 81 & 244 & 409 & 1.290 & 1.064 & 1.541 & 578 \\
\hline Comunidad Andina .................... & 5 & 79 & 15 & 14 & 114 & 33 & 90 \\
\hline 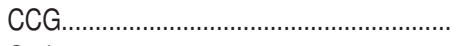 & 4.412 & 22 & 94 & 92 & 1.119 & 763 & 45 \\
\hline 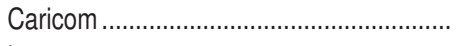 & 59 & 4 & 60 & 9 & 29 & 29 & 8 \\
\hline Latam & 270 & 750 & 923 & 3.227 & 1.949 & 2.194 & 960 \\
\hline 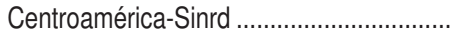 & 52 & 101 & 92 & 118 & 114 & 67 & 58 \\
\hline
\end{tabular}




\subsection{DISTRIBUCIÓN POR SECTORES DE ACTIVIDAD DEL FLUJO DE INVERSIÓN BRUTA ESPAÑOLA EN EL EXTERIOR (EXCLUYENDO LAS ETVE) \\ (Millones de euros)}

\begin{tabular}{|c|c|c|c|c|c|c|c|}
\hline & 2011 & 2012 & 2013 & 2014 & 2015 & 2016 & 2017 \\
\hline Agricultura, ganadería, caza y servicios relacionados con las mismas....... & 31 & 55 & 90 & 19 & 262 & 92 & 187 \\
\hline 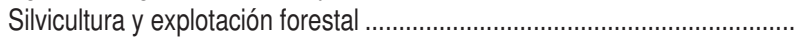 & 3 & 2 & 1 & 70 & 2 & 14 & 11 \\
\hline Pesca y acuicultura & 39 & 7 & 12 & 67 & 23 & 111 & 39 \\
\hline Extracción de crudo de petróleo y gas natural ..... & 578 & 795 & 669 & 2.477 & 782 & 877 & 1.256 \\
\hline 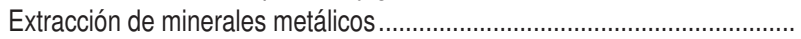 & 1 & 0 & - & - & - & 19 & 374 \\
\hline Otras industrias extractivas & 0 & 4 & 8 & 22 & 6 & 18 & 0 \\
\hline 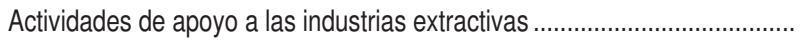 & 14 & 203 & 49 & 22 & 8.890 & 141 & 1.569 \\
\hline Industria de la alimentación & 220 & 185 & 206 & 582 & 355 & 262 & 160 \\
\hline Fabricación de bebidas & 28 & 278 & 70 & 39 & 6 & 57 & 20 \\
\hline Industria del tabaco & - & - & - & - & 0 & 4 & 1 \\
\hline Industria textil .................................. & 25 & 2 & 0 & 0 & 16 & 1 & 1 \\
\hline Confección de prendas de vestir & - & - & 1 & 0 & - & 0 & - \\
\hline 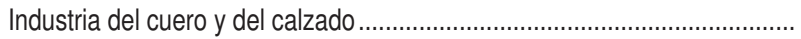 & 0 & 3 & 0 & - & 0 & 1 & - \\
\hline Industria madera y corcho, excepto muebles, cestería y espartería............ & - & - & 2 & 5 & 228 & 2 & - \\
\hline Industria del papel & 114 & 213 & 56 & 22 & 83 & 103 & 0 \\
\hline 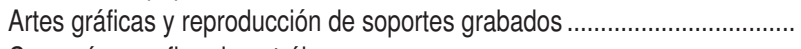 & 0 & 0 & - & - & 0 & 3 & 4 \\
\hline 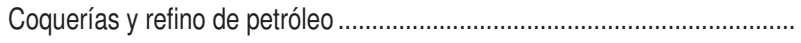 & 0 & - & 0 & 0 & - & - & 23 \\
\hline Industria química & 573 & 134 & 393 & 553 & 110 & 232 & 136 \\
\hline 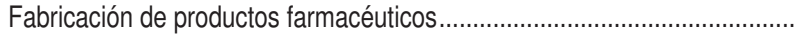 & 31 & 77 & 840 & 46 & 62 & 137 & 29 \\
\hline 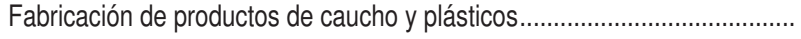 & 122 & 47 & 232 & 34 & 148 & 107 & 17 \\
\hline Fabricación de otros productos minerales no metálicos ............................... & 642 & 229 & 1.332 & 152 & 221 & 1.814 & 278 \\
\hline Metalurgia; fabricación productos hierro, acero y ferroaleaciones ............... & 1.268 & 936 & 699 & 415 & 32 & 39 & 66 \\
\hline Fabricación de productos metálicos, excepto maquinaria y equipo .............. & 89 & 90 & 52 & 63 & 329 & 86 & 62 \\
\hline Fabricación de productos informáticos, electrónicos y ópticos ..................... & 11 & 11 & 14 & 7 & 173 & 9 & 5 \\
\hline 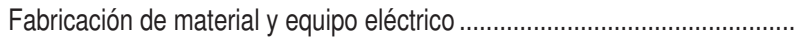 & 130 & 39 & 68 & 74 & 106 & 51 & 34 \\
\hline 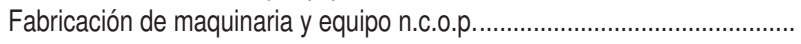 & 38 & 11 & 12 & 5 & 35 & 13 & 79 \\
\hline Fabricación de vehículos de motor, remolques y semirremolques .............. & 275 & 348 & 690 & 189 & 498 & 448 & 813 \\
\hline 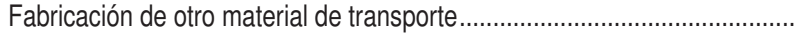 & 75 & 9 & 10 & 13 & 16 & 93 & 3 \\
\hline 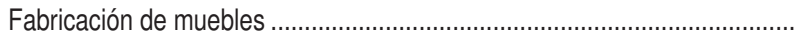 & 32 & 24 & 0 & 1 & 9 & 4 & 1 \\
\hline Otras industrias manufactureras & 6 & 21 & 27 & 18 & 11 & 46 & 8 \\
\hline 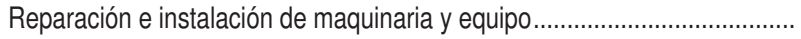 & 1 & 3 & 0 & 0 & 2 & 2.659 & 655 \\
\hline Suministro de energía eléctrica, gas, vapor y aire acondicionado ............... & 2.476 & 832 & 1.389 & 2.483 & 1.570 & 4.460 & 1.014 \\
\hline 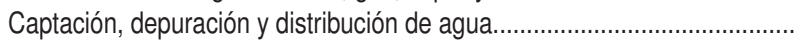 & 11 & 9 & 5 & 12 & 6 & 80 & 0 \\
\hline 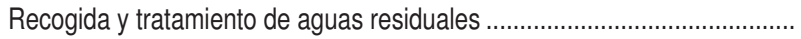 & 0 & 5 & - & - & - & 0 & - \\
\hline Recogida, tratamiento y eliminación de residuos; valorización ...................... & 24 & 54 & 34 & 1.573 & 107 & - & - \\
\hline Activid. de descontaminación y otros servicios de gestión de residuos....... & 2 & 0 & 0 & 4 & 20 & 53 & 0 \\
\hline 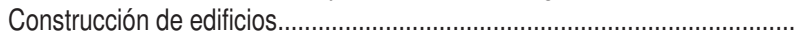 & 1.353 & 771 & 808 & 171 & 1.836 & 1.660 & 450 \\
\hline 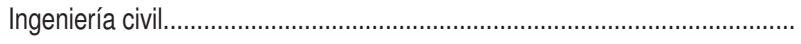 & 782 & 374 & 397 & 1.363 & 480 & 966 & 764 \\
\hline 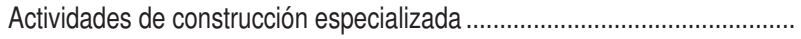 & 168 & 123 & 171 & 690 & 236 & 241 & 41 \\
\hline 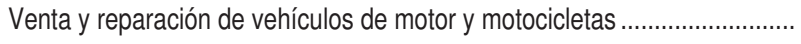 & 10 & 10 & 7 & 20 & 172 & 89 & 374 \\
\hline Comer. mayor e interme.comercio, excep. vehículos motor y motocicletas...... & 1.820 & 1.966 & 448 & 4.655 & 480 & 8.446 & 2.064 \\
\hline Comercio al por menor, excepto de vehículos de motor y motocicletas ......... & 558 & 678 & 271 & 255 & 256 & 369 & 120 \\
\hline 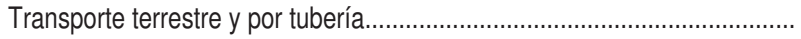 & 190 & 23 & 12 & 3 & 128 & 15 & 71 \\
\hline 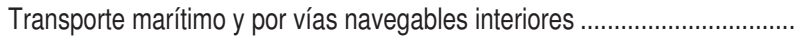 & 5 & 37 & 58 & 0 & 21 & 87 & - \\
\hline Transporte aéreo & 3.482 & 0 & 679 & 38 & 0 & 168 & 0 \\
\hline 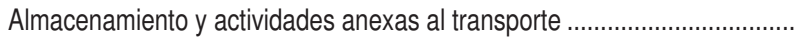 & 302 & 183 & 666 & 498 & 511 & 1.255 & 3.694 \\
\hline Actividades postales y de correos & - & - & - & - & - & - & 0 \\
\hline Servicios de alojamiento & 103 & 94 & 19 & 59 & 127 & 70 & 168 \\
\hline 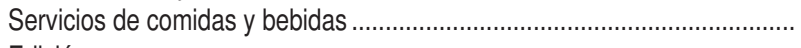 & 16 & 6 & 8 & 16 & 4 & 20 & 5 \\
\hline 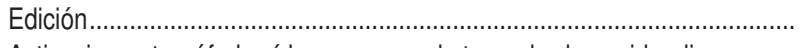 & 27 & 6 & 7 & 22 & 4 & 8 & 4 \\
\hline Activ. cinematográf, de vídeo y progra. de tv, grab. de sonid, edic mu....... & 37 & 26 & 79 & 3 & 139 & 19 & 3 \\
\hline Actividades de programación y emisión de radio y televisión ......................... & 7 & - & 0 & - & 16 & 109 & 33 \\
\hline 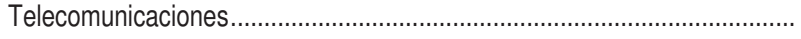 & 243 & 690 & 5.082 & 335 & 5.457 & 1.074 & 2.298 \\
\hline Programación,consultoría,otras actividades relacionadas informática......... & 91 & 1.891 & 142 & 102 & 136 & 850 & 39 \\
\hline Servicios de información & 19 & 8 & 14 & 225 & 7 & 9 & 1 \\
\hline 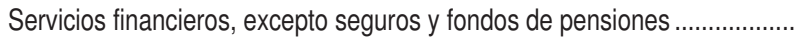 & 13.335 & 4.290 & 6.530 & 12.471 & 4.925 & 6.814 & 1.373 \\
\hline Seguros, reaseguros y fondos pensiones, excepto S. Social obligatoria.... & 2.440 & 462 & 1.510 & 657 & 1.020 & 302 & 493 \\
\hline
\end{tabular}


6.3. DISTRIBUCIÓN POR SECTORES DE ACTIVIDAD DEL FLUJO DE INVERSIÓN BRUTA ESPAÑOLA EN EL EXTERIOR (EXCLUYENDO LAS ETVE) (continuación)

(Millones de euros)

\begin{tabular}{|c|c|c|c|c|c|c|c|}
\hline & 2011 & 2012 & 2013 & 2014 & 2015 & 2016 & 2017 \\
\hline Actividades auxiliares a los servicios financieros y a los seguros................. & 319 & 241 & 674 & 217 & 107 & 81 & 185 \\
\hline 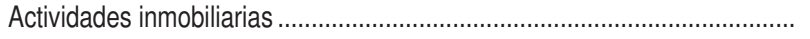 & 562 & 458 & 870 & 514 & 1.106 & 1.336 & 262 \\
\hline Actividades jurídicas y de contabilidad & 119 & 17 & 44 & 25 & 29 & 12 & 7 \\
\hline Actv. sedes centrales; activ. consultoría de gestión empresarial ................. & 237 & 82 & 101 & 66 & 36 & 83 & 42 \\
\hline Servicios tecnicos arquitectura e ingenieria, ensayos y analisis tecn .......... & 114 & 43 & 64 & 93 & 57 & 25 & 35 \\
\hline Investigación y desarrollo & 10 & 4 & 6 & 7 & 19 & 15 & 4 \\
\hline Publicidad y estudios de mercado & 0 & 0 & 66 & 28 & 10 & 2 & 9 \\
\hline 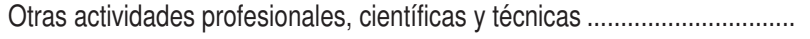 & 16 & 41 & 31 & 5 & 4 & 5 & 14 \\
\hline 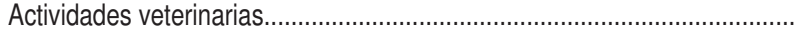 & 1 & 1 & 1 & 2 & - & - & - \\
\hline 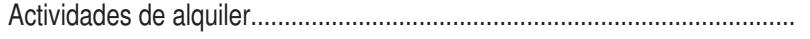 & 2 & 4 & 40 & 50 & 29 & 106 & 1 \\
\hline Actividades relacionadas con el empleo & 0 & - & 9 & 0 & 0 & 0 & 0 \\
\hline Activi. agencias viaje, operador turíst. reservas y acti. relacionadas ........... & 57 & 5 & 5 & 4 & 63 & 104 & 12 \\
\hline 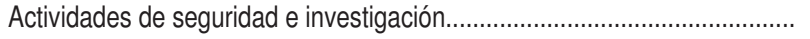 & 53 & 20 & 12 & 27 & 6 & 15 & 2 \\
\hline 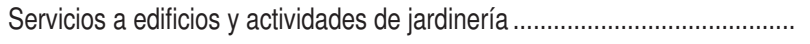 & 6 & 6 & 40 & 10 & 3 & 4 & 1 \\
\hline Actividades administrativas de oficina y otras auxiliares a empresas.......... & 13 & 172 & 7 & 13 & 49 & 92 & 70 \\
\hline 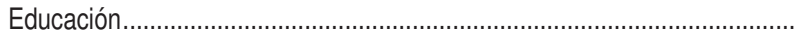 & 4 & 38 & 5 & 2 & 1 & 1 & 0 \\
\hline Actividades sanitarias & 4 & 179 & 9 & 17 & 7 & 10 & 207 \\
\hline 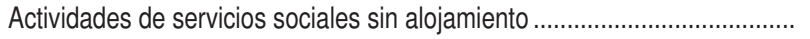 & - & 2 & - & 0 & 0 & - & - \\
\hline 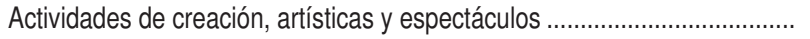 & - & - & - & - & - & 0 & - \\
\hline Activ. de bibliotecas, archivos, museos y otras activ. culturales ...................... & - & 0 & - & - & - & - & 1 \\
\hline 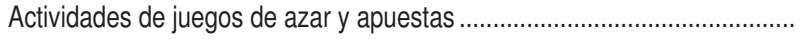 & 9 & 244 & 33 & 27 & 89 & 762 & 21 \\
\hline 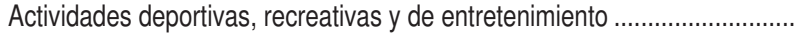 & 48 & 24 & 4 & 29 & 2 & 1 & 28 \\
\hline 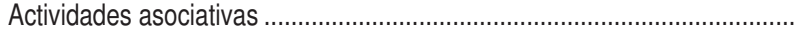 & 0 & 8 & 0 & 13 & 0 & 0 & 1 \\
\hline 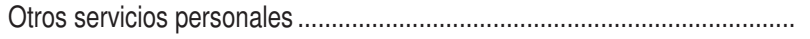 & 6 & 4 & 1 & 8 & 0 & 0 & 16 \\
\hline TOTAL GENERAL & 33.429 & 17.857 & 25.895 & 31.710 & 31.676 & 37.262 & 19.761 \\
\hline
\end{tabular}




\subsection{DISTRIBUCIÓN POR SECTORES DE ACTIVIDAD DEL FLUJO DE INVERSIÓN BRUTA EXTRANJERA EN ESPAÑA (EXCLUYENDO LAS ETVE) \\ (Millones de euros)}

\begin{tabular}{|c|c|c|c|c|c|c|c|}
\hline & 2011 & 2012 & 2013 & 2014 & 2015 & 2016 & 2017 \\
\hline Agricultura, ganadería, caza y servicios relacionados con las mismas......... & 75 & 31 & 72 & 419 & 521 & 339 & 310 \\
\hline 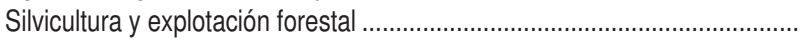 & 0 & 0 & 0 & 0 & 18 & - & 0 \\
\hline Pesca y acuicultura & 1 & 45 & 0 & 4 & 1 & 38 & 3 \\
\hline 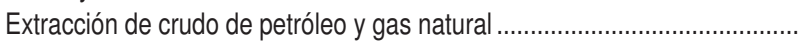 & 1 & 114 & 8 & 883 & 1 & 3 & 4 \\
\hline Extracción de minerales metálicos ........................ & 9 & 5 & 49 & 3 & 21 & 9 & 22 \\
\hline 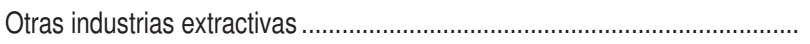 & 66 & 6 & 37 & 206 & 4 & 62 & 0 \\
\hline 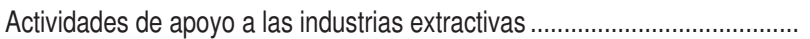 & 1 & 23 & 40 & 5 & 0 & 0 & 3 \\
\hline 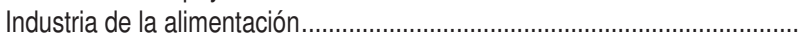 & 689 & 325 & 164 & 801 & 1.166 & 715 & 633 \\
\hline 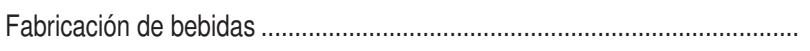 & 71 & 43 & 27 & 204 & 157 & 18 & 73 \\
\hline Industria del tabaco & - & 0 & 2 & 0 & - & - & 2 \\
\hline 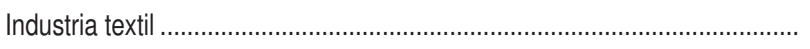 & 19 & 8 & 8 & 4 & 15 & 11 & 10 \\
\hline 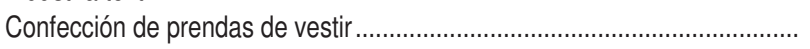 & 11 & 8 & 12 & 2 & 8 & 1 & 189 \\
\hline Industria del cuero y del calzado & 16 & 1 & 3 & 1 & 8 & 0 & 1 \\
\hline Industria madera y corcho, excepto muebles, cestería y espartería............. & 33 & 5 & 35 & 90 & 160 & 232 & 3 \\
\hline Industria del papel & 42 & 307 & 117 & 10 & 67 & 13 & 14 \\
\hline 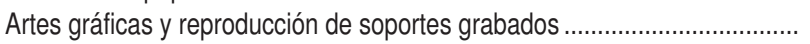 & 17 & 7 & 20 & 6 & 74 & 3 & 0 \\
\hline Coquerías y refino de petróleo & 4.195 & - & - & - & 0 & - & - \\
\hline Industria química & 259 & 479 & 72 & 484 & 219 & 203 & 319 \\
\hline 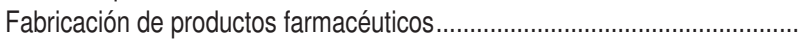 & 98 & 348 & 787 & 234 & 133 & 80 & 212 \\
\hline 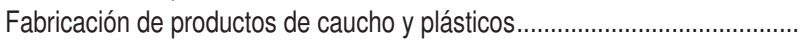 & 31 & 130 & 45 & 19 & 484 & 88 & 168 \\
\hline 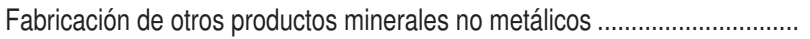 & 851 & 603 & 161 & 164 & 16 & 798 & 1.155 \\
\hline Metalurgia; fabricación productos hierro, acero y ferroaleaciones................ & 1.084 & 1.430 & 661 & 39 & 80 & 591 & 455 \\
\hline Fabricación de productos metálicos, excepto maquinaria y equipo ............... & 256 & 54 & 178 & 20 & 52 & 438 & 40 \\
\hline Fabricación de productos informáticos, electrónicos y ópticos ..................... & 67 & 3 & 35 & 5 & 100 & 137 & 82 \\
\hline 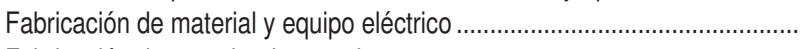 & 198 & 116 & 122 & 43 & 26 & 163 & 208 \\
\hline 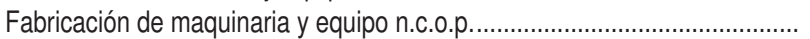 & 61 & 151 & 79 & 34 & 60 & 45 & 264 \\
\hline Fabricación de vehículos de motor, remolques y semirremolques ............... & 163 & 732 & 347 & 68 & 247 & 439 & 204 \\
\hline 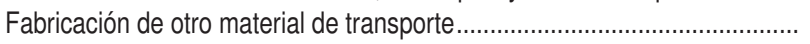 & 23 & 1 & 17 & 3 & 115 & 771 & 5 \\
\hline Fabricación de muebles & 7 & 0 & 0 & 0 & 2 & 11 & 14 \\
\hline Otras industrias manufactureras & 10 & 5 & 1 & 1 & 121 & 2 & 12 \\
\hline 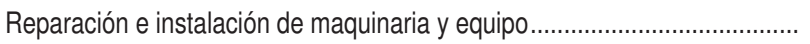 & 10 & 14 & 8 & 82 & 6 & 6 & 1 \\
\hline Suministro de energía eléctrica, gas, vapor y aire acondicionado ................ & 1.663 & 1.218 & 1.548 & 1.014 & 2.903 & 4.394 & 2.441 \\
\hline 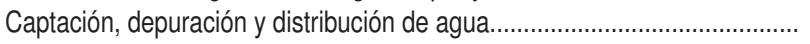 & 16 & 59 & 0 & 455 & 2 & 0 & 600 \\
\hline 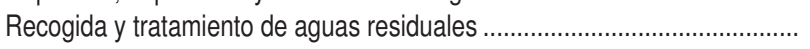 & 0 & 0 & 0 & 0 & 0 & 0 & 15 \\
\hline Recogida, tratamiento y eliminación de residuos; valorización ..................... & 1 & 5 & 158 & 0 & 8 & 4 & 2 \\
\hline Activid. de descontaminación y otros servicios de gestión de residuos........ & 0 & 2 & 625 & 0 & 3 & 0 & 0 \\
\hline 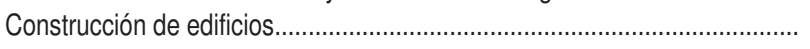 & 703 & 517 & 614 & 1.123 & 4.587 & 1.473 & 1.144 \\
\hline 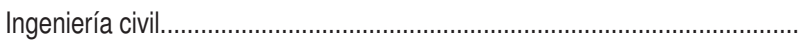 & 9 & 238 & 33 & 554 & 81 & 1.074 & 1.003 \\
\hline 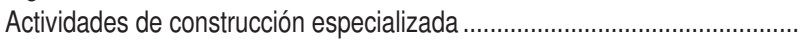 & 257 & 376 & 850 & 103 & 165 & 52 & 42 \\
\hline Venta y reparación de vehículos de motor y motocicletas .............................. & 177 & 65 & 28 & 23 & 214 & 197 & 208 \\
\hline Comer. mayor e interme.comercio, excep. vehículos motor y motocicletas......... & 623 & 489 & 741 & 3.439 & 1.508 & 1.081 & 1.519 \\
\hline Comercio al por menor, excepto de vehículos de motor y motocicletas ....... & 379 & 286 & 220 & 301 & 874 & 444 & 197 \\
\hline 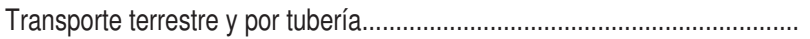 & 146 & 27 & 739 & 109 & 330 & 529 & 638 \\
\hline 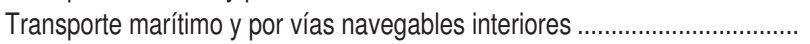 & 21 & 23 & 12 & 4 & 3 & 0 & 204 \\
\hline Transporte aéreo & 3.960 & 35 & 116 & 0 & 28 & 679 & 38 \\
\hline 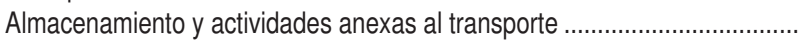 & 454 & 80 & 290 & 646 & 559 & 571 & 258 \\
\hline 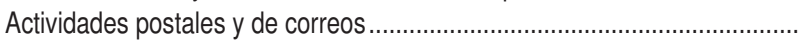 & 3 & 5 & 2 & 1 & 0 & 3 & 0 \\
\hline Servicios de alojamiento & 281 & 101 & 379 & 747 & 223 & 345 & 253 \\
\hline Servicios de comidas y bebidas & 53 & 20 & 153 & 445 & 308 & 310 & 43 \\
\hline 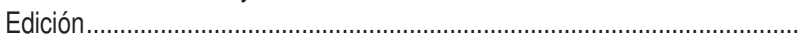 & 30 & 10 & 181 & 7 & 97 & 103 & 51 \\
\hline Activ. cinematográf, de vídeo y progra. de tv, grab. de sonid, edic mu........ & 290 & 42 & 30 & 4 & 150 & 69 & 24 \\
\hline Actividades de programación y emisión de radio y televisión ....................... & 1 & 14 & 23 & 0 & 0 & 0 & 10 \\
\hline 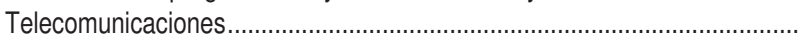 & 4.747 & 401 & 392 & 353 & 89 & 52 & 1.586 \\
\hline Programación,consultoría,otras actividades relacionadas informática.......... & 59 & 173 & 153 & 411 & 141 & 277 & 273 \\
\hline Servicios de información & 35 & 29 & 24 & 25 & 53 & 264 & 23 \\
\hline Servicios financieros, excepto seguros y fondos de pensiones ..................... & 3.102 & 1.669 & 913 & 1.885 & 471 & 2.271 & 1.670 \\
\hline Actividades de las sociedades holding & 68 & 91 & 14 & 2 & 42 & 88 & 1 \\
\hline
\end{tabular}




\subsection{DISTRIBUCIÓN POR SECTORES DE ACTIVIDAD DEL FLUJO DE INVERSIÓN BRUTA EXTRANJERA EN ESPAÑA} (EXCLUYENDO LAS ETVE) (continuación)

(Millones de euros)

\begin{tabular}{|c|c|c|c|c|c|c|c|}
\hline & 2011 & 2012 & 2013 & 2014 & 2015 & 2016 & 2017 \\
\hline Seguros, reaseguros y fondos pensiones, excepto S. Social obligatoria..... & 590 & 165 & 1.195 & 56 & 20 & 46 & 535 \\
\hline Actividades auxiliares a los servicios financieros y a los seguros................. & 189 & 327 & 985 & 666 & 1.591 & 710 & 351 \\
\hline 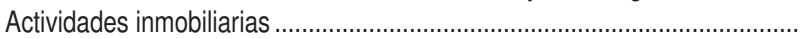 & 1.411 & 1.091 & 1.879 & 2.981 & 4.202 & 3.414 & 3.131 \\
\hline 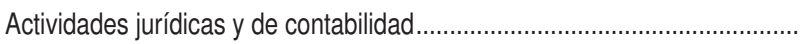 & 43 & 90 & 62 & 22 & 144 & 164 & 150 \\
\hline Actv. sedes centrales; activ. consultoría de gestión empresarial ................. & 72 & 42 & 59 & 43 & 161 & 150 & 704 \\
\hline Actividades de las sedes centrales & 0 & 1 & 0 & 0 & 29 & 38 & 2 \\
\hline Servicios tecnicos arquitectura e ingenieria, ensayos y analisis tecn ........... & 281 & 428 & 231 & 138 & 64 & 484 & 41 \\
\hline Investigación y desarrollo & 9 & 10 & 8 & 36 & 33 & 124 & 71 \\
\hline Publicidad y estudios de mercado & 30 & 68 & 33 & 14 & 118 & 56 & 404 \\
\hline 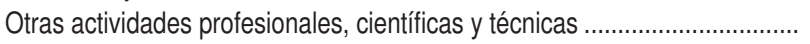 & 39 & 8 & 7 & 47 & 12 & 31 & 69 \\
\hline 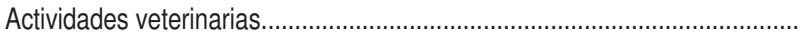 & - & 0 & - & - & 0 & 5 & 0 \\
\hline Actividades de alquiler................................. & 18 & 222 & 156 & 83 & 50 & 70 & 388 \\
\hline 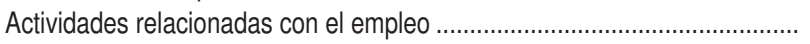 & 31 & 40 & 82 & 1 & 9 & 0 & 5 \\
\hline Activi. agencias viaje, operador turíst. reservas y acti. relacionadas ............ & 169 & 53 & 19 & 48 & 5 & 9 & 40 \\
\hline Actividades de seguridad e investigación & 2 & 2 & 1 & 0 & 1 & 4 & 1 \\
\hline 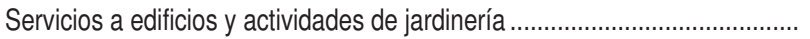 & 1 & 2 & 1 & 0 & 21 & 30 & 1 \\
\hline Actividades administrativas de oficina y otras auxiliares a empresas ........... & 32 & 561 & 91 & 119 & 71 & 123 & 82 \\
\hline Administración Pública y defensa; Seguridad Social obligatoria.................. & 0 & 0 & 0 & 4 & - & - & - \\
\hline Educación & 0 & 85 & 53 & 120 & 882 & 17 & 39 \\
\hline Actividades sanitarias & 31 & 350 & 48 & 83 & 211 & 148 & 628 \\
\hline 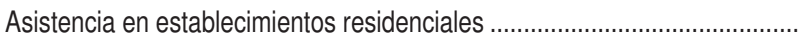 & 55 & 2 & 15 & 0 & 108 & 48 & 87 \\
\hline 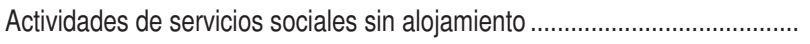 & 0 & 21 & 3 & 6 & 0 & 209 & 1 \\
\hline 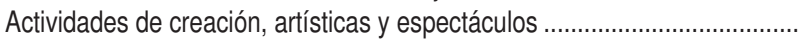 & 1 & 0 & 18 & 10 & 5 & 9 & 31 \\
\hline Activ. de bibliotecas, archivos, museos y otras activ. culturales .................. & 0 & 0 & 1 & 4 & 0 & 1 & 0 \\
\hline 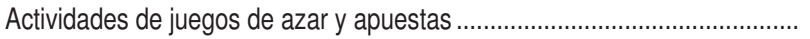 & 10 & 2 & 15 & 7 & 54 & 12 & 0 \\
\hline 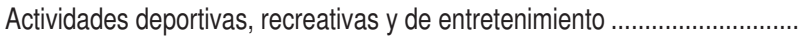 & 264 & 179 & 950 & 156 & 233 & 181 & 178 \\
\hline 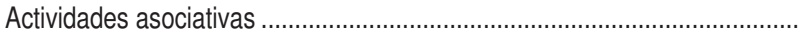 & 0 & 0 & 13 & 0 & 0 & 4 & 0 \\
\hline Reparación ordenadores, efectos personales y artículos uso doméstico .... & 1 & 0 & - & 0 & 0 & 0 & 0 \\
\hline Otros servicios personales & 11 & 8 & 0 & 252 & 41 & 18 & 164 \\
\hline Actividades de los hogares como empleadores de personal doméstico...... & - & - & - & 0 & - & - & 0 \\
\hline Activ. hogares como produc. bienes y serv para uso propio ......................... & - & - & - & - & - & 0 & - \\
\hline Actividades de organizaciones y organismos extraterritoriales....................... & - & - & - & - & - & - & - \\
\hline 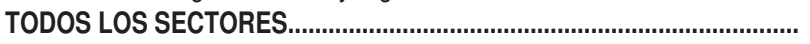 & 28.732 & 14.729 & 17.271 & 20.380 & 24.785 & 25.602 & 23.758 \\
\hline
\end{tabular}

